\title{
DHEA supplementation can result in assay changes which may impact clinical decisions in IVF
}

\author{
Jason Franasiak $^{1,2} \cdot$ Eric Forman $^{1,2} \cdot$ Richard Scott $^{1,2}$
}

Received: 31 March 2017 / Accepted: 13 April 2017 /Published online: 29 April 2017

(C) Springer Science+Business Media New York 2017

Dear Editor,

We read with interest Tsui et al.'s letter to the editor regarding DHEA-S interference with progesterone assays in reference to our manuscript. In it, they pointed out that the units for DHEA-S should have been micrograms per deciliter as noted in Fig. 2 of the manuscript. The micrograms per deciliter units should have been used throughout the manuscript and the use of micrograms per milliliter was an error, both in the scientific abstract and in the manuscript. DHEA-S controls from the manufacturer were supplied in micrograms per deciliter units and yielded assay results in the reportable range in micrograms per deciliter. With the proper units of measurement, the DHEA-S levels measured in our patient population, both those taking DHEA supplementation and controls, were within physiological ranges. We appreciate their careful review of the manuscript and appreciate the opportunity to correct this error.

The authors repeated the experiment on their Siemens ADVIA Centaur assay and showed an increase in measured progesterone from 0.4 to $1.0 \mathrm{ng} / \mathrm{mL}$ which was a similar, but less pronounced, change noted in our manuscript in Fig. 1. There was a greater change noted on the progesterone assay of other platforms.

The interactions reported with the corrected units of micrograms per deciliter rather than micrograms per milliliter remain true. Tsui et al.'s experiment lends support to the theory that circulating DHEA-S in women on supplementation may alter serum progesterone levels. Although the interactions may be small in absolute units, when clinical decisions regarding whether to cryopreserve all embryos rather than perform a fresh embryo transfer due to premature progesterone rise are in the balance, slight changes may have a meaningful clinical impact.

Sincerely,

Jason M. Franasiak, MD, TS

Eric J. Forman, MD, HCLD/ALD

Richard T. Scott, MD, HCLD/ALD

This reply refers to the comment available at doi:10.1007/s10815-0170921-7

Jason Franasiak

jfranasiak@rmanj.com

1 Reproductive Medicine Associates of New Jersey, Basking Ridge, NJ, USA

2 Thomas Jefferson University, Philadelphia, PA, USA 